PROCEEDINGS OF THE

AMERICAN MATHEMATICAL SOCIETY

Volume 128, Number 10, Pages 3099-3106

S 0002-9939(00)05353-3

Article electronically published on April 28, 2000

\title{
THE MARDEŠIĆ FACTORIZATION THEOREM FOR EXTENSION THEORY AND C-SEPARATION
}

\author{
MICHAEL LEVIN, LEONARD R. RUBIN, AND PHILIP J. SCHAPIRO \\ (Communicated by Alan Dow)
}

\begin{abstract}
We shall prove a type of Mardešić factorization theorem for extension theory over an arbitrary stratum of $\mathrm{CW}$-complexes in the class of arbitrary compact Hausdorff spaces. Our result provides that the space through which the factorization occurs will have the same strong countability property (e.g., strong countable dimension) as the original one had. Taking into consideration the class of compact Hausdorff spaces, this result extends all previous ones of its type. Our factorization theorem will simultaneously include factorization for weak infinite-dimensionality and for Property C, that is, for C-spaces.

A corollary to our result will be that for any weight $\alpha$ and any finitely homotopy dominated CW-complex $K$, there exists a Hausdorff compactum $X$ with weight $w X \leq \alpha$ and which is universal for the property $X \tau K$ and weight $\leq \alpha$. The condition $X \tau K$ means that for every closed subset $A$ of $X$ and every map $f: A \rightarrow K$, there exists a map $F: X \rightarrow K$ which is an extension of $f$. The universality means that for every compact Hausdorff space $Y$ whose weight is $\leq \alpha$ and for which $Y \tau K$ is true, there is an embedding of $Y$ into $X$.

We shall show, on the other hand, that there exists a CW-complex $S$ which is not finitely homotopy dominated but which has the property that for each weight $\alpha$, there exists a Hausdorff compactum which is universal for the property $X \tau S$ and weight $\leq \alpha$.
\end{abstract}

\section{INTRODUCTION}

In this paper we shall prove the following result.

1.1. Theorem (Factorization Theorem for Extension Theory and C-Separation). Let $f: X \rightarrow Y$ be a map of compact Hausdorff spaces $X$ and $Y$, and suppose that $\mathcal{A}$ is a countable collection of closed subsets of $X$. Then there exists a compact Hausdorff space $Z$, a surjective map $g: X \rightarrow Z$, and a map $p: Z \rightarrow Y$ such that

(a) $w Z \leq \max \left\{w Y, \aleph_{0}\right\}$,

(b) $p \circ g=f$,

(c) if $N$ is a $C W$-complex, $D \in \mathcal{A}, A$ is a closed subset of $g(D), f_{0}: A \rightarrow N$ is a map, and there exists a map $F: D \rightarrow N$ such that $F(x)=f_{0}(g(x))$ for all

Received by the editors March 19, 1998 and, in revised form, November 13, 1998.

1991 Mathematics Subject Classification. Primary 54F45, 55M10.

Key words and phrases. Cohomological dimension, covering dimension, extension theory, inverse sequences, inverse systems, inverse limits, compactification, Mardešić factorization, weight, universal compactum, homotopy domination, C-space, weak infinite-dimension, strong countabledimension.

A portion of this work was completed while the first-named author was a J. Clarence Karcher Visitor in the Department of Mathematics at the University of Oklahoma. 
$x \in g^{-1}(A) \cap D$, then there is a map $H: g(D) \rightarrow N$ which is an extension of $f_{0}$, and

(d) if $\mathcal{S}$ is a sequence of open covers of $Z, D \in \mathcal{A}$, and $g^{-1}(\mathcal{S}) \mid D$ has a $C$ refinement in $D$, then $\mathcal{S} \mid g(D)$ has a C-refinement in $g(D)$.

We shall provide a proof in section 2 .

1.2. Definition. Whenever $X, Y, Z$ are compact Hausdorff spaces, $f: X \rightarrow Y$, $g: X \rightarrow Z, p: Z \rightarrow Y$ are maps, $g$ is surjective, and $p \circ g=f$, we shall refer to $(g, p)$ as a factorization of $f$ through $Z$.

By definition, for a space $X$ and a CW-complex $K, X \tau K$ (sometimes written $\operatorname{dim} X \leq K)$ means that for each closed subset $A$ of $X$ and map $f: A \rightarrow K$, there exists a map $F: X \rightarrow K$ which is an extension of $f$. (By map, we always mean continuous function). This is the basic notion of extension theory [DD]. Each dimension theory, covering dimension dim or cohomological dimension $\operatorname{dim}_{G}$ over an abelian group $G$, can be expressed in terms of extension theory. For it is well known ([Na]) that for paracompact spaces $X$, $\operatorname{dim} X \leq n$ if and only if $X \tau S^{n}$ and that $\operatorname{dim}_{G} X \leq n$ (for an arbitrary abelian group $G$ ) if and only if $X \tau K$ for each Eilenberg-MacLane complex $K$ of type $K(G, n)$.

Theorem 1.1 leads to the following corollary which captures the original Mardešić Factorization Theorem ([Ma1] ) as well as all of its direct successors for the class of compact Hausdorff spaces.

1.3. Corollary. Let $f: X \rightarrow Y$ be a map of compact Hausdorff spaces, $(g, p)$ be a factorization of $f$ through a compact Hausdorff space $Z$ with $w Z \leq \max \left\{w Y, \aleph_{0}\right\}$ as in Theorem 1.1, and assume that $X \in \mathcal{A}$. If $K$ is an arbitrary $C W$-complex and $X \tau K$, then $Z \tau K$. Consequently, if $\operatorname{dim} X \leq n$, then $\operatorname{dim} Z \leq n$; if $G$ is an abelian group and $\operatorname{dim}_{G} X \leq n$, then $\operatorname{dim}_{G} Z \leq n$.

The first version of the Mardešić Factorization Theorem appeared in Ma1 where it was designed for dimension theory dim (see also [Ma2]). The work in [Pa] (Corollary 2) improves that in Ma1, yielding a theorem like our Corollary 1.3 but with the restriction that $w K \leq w Y$ (see also [Dr], page 472, Factorization Theorem). Proposition 4.9 of $\mathrm{Ch}$ contains a factorization theorem for Tychonoff spaces which uses as "complexes", ANR Polish spaces. Since every countable CW-complex is homotopy equivalent to a Polish ANR, then that result when restricted to the class of compact Hausdorff spaces contains the result of [Dr], at least for countable complexes. We refer the reader to section 2 of Dy1 for several results concerning factorization in the class of separable metrizable spaces. Other authors such as A. Arhangel'skiı̌, W. Luxemburg and A. Zarelua have made significant contributions to this subject.

A nice aspect of our factorization is that it can preserve a property such as strong countable dimension. Let us state a corollary which makes this explicit.

1.4. Corollary. Let $f: X \rightarrow Y$ be a map of compact Hausdorff spaces, and assume that $X_{i}$ are closed subspaces of $X$ for each $i \in \mathbb{N}$. Then there is a factorization $(g, p)$ of $f$ through a compact Hausdorff space $Z$ with $w Z \leq \max \left\{w Y, \aleph_{0}\right\}$ such that if $K$ is a $C W$-complex and $X_{i} \tau K$, then $g\left(X_{i}\right) \tau K$. In the notation of [DD], this could be stated as

$$
\operatorname{ext}-\operatorname{dim}\left(g\left(X_{i}\right)\right) \leq \operatorname{ext}-\operatorname{dim}\left(X_{i}\right)
$$


The notions, weak infinite-dimensionality and C-space (called Property $\mathrm{C}$ in some references) are defined in En2. For compact Hausdorff spaces, as required herein, there is a more concise, unified approach to these concepts.

1.5. Definition. Let $\mathcal{S}=\left\{\mathcal{S}_{i} \mid i \in \mathbb{N}\right\}$ be a sequence of families of subsets of a space $X$. Then we say that $\mathcal{S}$ is finite if for almost all $i, \mathcal{S}_{i}=\emptyset$. A sequence $\mathcal{C}=\left\{\mathcal{C}_{i} \mid i \in \mathbb{N}\right\}$ of families of subsets of $X$ refines $\mathcal{S}$ if $\mathcal{C}_{i}$ refines $\mathcal{S}_{i}$ for each $i$, and is called a C-refinement if in addition each $\mathcal{C}_{i}$ is closed and discrete and $\bigcup \mathcal{C}$ covers $X$.

The reader should be able to construct a proof of the next statement.

1.6. Lemma. A compact Hausdorff space $X$ is a $C$-space (weakly infinite-dimensional space) if every sequence of open (two-element open) covers of $X$ admits a C-refinement.

This yields a third corollary to Theorem 1.1.

1.7. Corollary. Let $f: X \rightarrow Y$ and $Z$ be as in Theorem 1.1 and assume that $X \in \mathcal{A}$. If $X$ is a $C$-space, then so is $Z$. If $X$ is weakly infinite-dimensional, then so is $Z$.

1.8. Definition. Let $K$ be a CW-complex, $\mathcal{C}$ a class of spaces, and $X \in \mathcal{C}$. Then we say that $X$ is universal for $K$-extension theory, if $X \tau K$, and for each $Y \in \mathcal{C}$ with $Y \tau K$, there is an embedding of $Y$ into $X$.

The next result can be obtained from previous work such as $\mathrm{Dr}$ (see also Dy2], DM] for related material), but it also follows readily from Theorem 1.1, so we will indicate a proof here. The existence (or lack of existence) of universal compacta even in case $K$ is of type $K(\mathbb{Z}, n)$ and $\alpha=\aleph_{0}$ is not known.

1.9. Corollary. For every finitely homotopy dominated $C W$-complex $K$ and every infinite cardinal $\alpha$, there exists a compact Hausdorff space $X$ which is universal for the class of Tychonoff spaces of weight $\leq \alpha$ and $K$-extension theory.

Proof. Let us use $\beta Y$ to designate the Stone-Čech compactification of a Tychonoff space $Y$. It is easy to check that if $K$ is a CW-complex and the space $Y$ is the topological sum of a collection $\left\{Y_{a} \mid a \in A\right\}$ of Tychonoff spaces with $Y_{a} \tau K$ for each $a \in A$, then $Y$ is a Tychonoff space and $Y \tau K$. The result now follows from application of Lemma 1.10 below, 7.4 .14 and 7.4.16 of [En1] and Theorem 1.1.

1.10. Lemma. Let $K$ be a finitely homotopy dominated $C W$-complex and let $Y$ be a Tychonoff space such that $Y \tau K$. Then $\beta Y \tau K$.

Proof. Let $A \subset \beta Y$ be closed and $f: A \rightarrow K$ be a map. Applying Lemma 2.11 of [RS, one may find a closed neighborhood $N$ of $A$ in $\beta Y$ such that $\mathrm{Cl}_{\beta Y}(N \cap Y)=N$ and $f$ extends to a map $f_{0}: N \rightarrow K$. Since $Y \tau K$, there exists a map $F: N \cup Y \rightarrow K$ such that $F \mid N=f_{0}: N \rightarrow K$. Since $K$ is finitely dominated, $F$ is homotopic to a map $G: N \cup Y \rightarrow K$ where $G(N \cup Y) \subset K_{0} \subset K$ and $K_{0}$ is compact. Let $H: \beta Y \rightarrow K_{0}$ be the unique continuous extension of $G \mid Y: Y \rightarrow K_{0}$. Then of course $G|A=H| A: A \rightarrow K_{0} \subset K$. Since $f=F|A \simeq G| A=H \mid A$ and $H \mid A$ extends to $H: \beta Y \rightarrow K$, then the homotopy extension property shows us that $f$ extends to a map of $\beta Y$ to $K$. 
To help round out the discussion, let us give a simple example.

1.11. Lemma. There exists a $C W$-complex $S$ which is not finitely homotopy dominated, but which has the property that for each weight $\alpha$, there is a Hausdorff compactum $X$ which is universal for $S$-extension theory, and compact Hausdorff spaces of weight $\leq \alpha$.

Proof. Define $S=[1 / 2, \infty) \cup \bigcup_{i=1}^{\infty} S_{i} \subset \mathbb{R}^{2}$ where $S_{i}$ is the circle centered at $(i, 1 / 2) \in$ $\mathbb{R}^{2}$ whose radius is $1 / 2$. Then $S$ has the structure of a connected CW-complex and $\pi_{1}(S)$ is free on a countably infinite set of generators. One can see from this fact that $S$ is not finitely homotopy dominated.

Since $S^{1}$ is homeomorphic to a retract of $S$, then it is easy to detect that $X \tau S$ implies $X \tau S^{1}$. Conversely, suppose $X$ is a compact Hausdorff space and $X \tau S^{1}$. Then $\operatorname{dim} X \leq 1$. From this perspective it is not difficult to see that any map of a closed subset of $X$ to a connected CW-complex $K$ extends to a map of $X$ to $K$. Hence $X \tau S$.

We have demonstrated that for any compact Hausdorff space $X, X \tau S^{1}$ if and only if $X \tau S$. By Corollary 1.9, for any $\alpha$ there exists a Hausdorff compactum which is universal for $S$-extension theory and weight $\alpha$. (Of course, $X \tau S^{1}$ simply means that $\operatorname{dim} X \leq 1$, and the existence of universal compacta for dim has long been known [En1].)

Every CW-complex $K_{0}$ is homotopy equivalent to $|K|$ for some simplicial complex $K$ (all polyhedra $|K|$ are given the weak topology herein). Since it is convenient for us to work in the class of simplicial complexes and their polyhedra, all our proofs in section 2 will be in terms of these objects instead of CW-complexes.

The authors are thankful for valuable suggestions given to us by A. Dranishnikov and for the thorough effort and useful recommendations of our referee.

\section{Proof of the Factorization Theorem}

We first make a definition which will be convenient for our work below.

2.1. Definition. Let $X, Y, Z$ be spaces, $D$ a subset of $X$, and suppose we are given maps $f: X \rightarrow Y, g: X \rightarrow Z$, and $p: Z \rightarrow Y$ such that $p \circ g=f$. Then we shall say that the triple $(f, g, p)$ is lifting for simplicial complexes relative to $D$ if for each closed subset $A$ of $Y$, each simplicial complex $N$ and each pair of maps $h: A \rightarrow|N|, F: D \rightarrow|N|$ having the property that $F(x)=h(f(x))$ for all $x \in D \cap f^{-1}(A)$, there exists a map $H: g(D) \rightarrow|N|$ such that

$$
H(z)=h(p(z)) \text { for all } z \in g(D) \cap p^{-1}(A) .
$$

We call $(f, g, p)$ C-lifting relative to $D$ if for each sequence $\mathcal{S}$ of open covers of $Y$ such that the sequence $f^{-1}(\mathcal{S}) \mid D$ has a C-refinement in $D$, the sequence $p^{-1}(\mathcal{S}) \mid g(D)$ has a C-refinement in $g(D)$.

Our proof of Theorem 1.1 is based on the next lemma whose proof will be provided later.

2.2. Lemma. Let $X, Z_{1}$ be compact Hausdorff spaces, $D \subset X$ be closed, and $f_{1}: X \rightarrow Z_{1}$ be a map. Then there exists a compact Hausdorff space $Z_{2}$ with $w Z_{2} \leq \max \left\{w Z_{1}, \aleph_{0}\right\}$, a surjective map $f_{2}: X \rightarrow Z_{2}$, and a map $p: Z_{2} \rightarrow Z_{1}$ such that $p \circ f_{2}=f_{1}$ and $\left(f_{1}, f_{2}, p\right)$ is simultaneously lifting for simplicial complexes relative to $D$ and $C$-lifting relative to $D$. 
We shall also use the next two facts; but we leave their proofs to the reader.

2.3. Lemma. Let $g: X \rightarrow Z$ be a map of compact Hausdorff spaces, $N$ be a finite simplicial complex, $A$ be a closed subspace of $Z$, and $f_{0}: A \rightarrow|N|, F: X \rightarrow|N|$ be maps such that $F(x)=f_{0}(g(x))$ for all $x \in g^{-1}(A)$. Then there exists a closed neighborhood $B$ of $A$ in $Z$ and a map $\tilde{f}_{0}: B \rightarrow|N|$ extending $f_{0}$, such that, with $B_{0}=g^{-1}(B), F\left|B_{0}: B_{0} \rightarrow\right| N \mid$ is homotopic to $\tilde{f}_{0} \circ g\left|B_{0}: B_{0} \rightarrow\right| N \mid$.

2.4. Lemma. Let $X$ be a compact Hausdorff space and let $\mathcal{S}$ be a sequence of open covers of $X$ which has a $C$-refinement in $X$. Then $\mathcal{S}$ has a $C$-refinement in $X$ which is finite.

Proof of Theorem 1.1. Let $Z_{1}=f(X), f_{1}=f: X \rightarrow Z_{1}$, and write $\mathcal{A}=\left\{D_{1}, D_{2}\right.$, $\ldots\}$. Let $\gamma: \mathbb{N} \rightarrow \mathbb{N}$ be a surjection each of whose point inverses is infinite. Apply Lemma 2.2 recursively to obtain a sequence $\left(Z_{i}\right)_{i=1}^{\infty}$ of compact Hausdorff spaces, two sequences $\left(f_{i}: X \rightarrow Z_{i}\right)_{i=1}^{\infty},\left(p_{i i+1}: Z_{i+1} \rightarrow Z_{i}\right)_{i=1}^{\infty}$ of maps, the $f_{i}$ 's being surjective, such that for each $i$,

(1) $w Z_{i+1} \leq \max \left\{w Z_{i}, \aleph_{0}\right\}$

(2) $p_{i i+1} \circ f_{i+1}=f_{i}$, and

(3) $\left(f_{i}, f_{i+1}, p_{i i+1}\right)$ is lifting for simplicial complexes relative to $D_{\gamma(i)}$ and simultaneously is C-lifting relative to $D_{\gamma(i)}$.

Put $Z=\lim \left(Z_{i}, p_{i+1}, \mathbb{N}\right)$. Then $Z$ is a compact Hausdorff space and (1) shows that $w Z \leq \max \left\{w Y, \aleph_{0}\right\}$. Using (2), let $g: X \rightarrow Z$ be the limit of the compatible system of surjective maps $f_{i}: X \rightarrow Z_{i}$. Then $g$ is easily seen to be surjective. Let $p_{i}: Z \rightarrow Z_{i}$ denote coordinate projection, and define $p=p_{1}: Z \rightarrow Z_{1} \subset Y$. Then of course, $p \circ g=f$. Fix $D=D_{k} \in \mathcal{A}$ and let $D_{0}=g(D)$, and $g_{0}=g \mid D: D \rightarrow D_{0}$.

We want to show that (c) of 1.1 is true. Let $A$ be a closed subset of $D_{0}, N_{0}$ be a CW-complex, and $f_{0}: A \rightarrow N_{0}, F: D \rightarrow N_{0}$ be maps such that $F(x)=f_{0}(g(x))=$ $f_{0}\left(g_{0}(x)\right)$ for all $x \in g^{-1}(A) \cap D=g_{0}^{-1}(A)$. The reader will easily see that there is no loss of generality in replacing $N_{0}$ by $|N|$, where $N$ is a finite simplicial complex. We have to prove the existence of a map of $D_{0}$ to $|N|$ which is an extension of $f_{0}$.

Using Lemma 2.3, extend $f_{0}$ to a map $\tilde{f}_{0}: B \rightarrow|N|$, where $B$ is a closed neighborhood of $A$ in $D_{0}$ in such a manner that $F\left|B_{0}: B_{0} \rightarrow\right| N \mid$ is homotopic to $\tilde{f}_{0} \circ g_{0}\left|B_{0}: B_{0} \rightarrow\right| N \mid$, where $B_{0}=g_{0}^{-1}(B)$.

One may choose $j$ so large that for each $i \geq j, p_{i}^{-1}\left(p_{i}(A)\right) \cap D_{0} \subset B$. Hence there exists $i$ such that $k=\gamma(i)$, and a map $h: p_{i}(B) \rightarrow|N|$ such that $h \circ p_{i} \mid B \simeq \tilde{f}_{0}$ and $\tilde{A}=p_{i}^{-1}\left(p_{i}(A)\right) \cap D_{0} \subset B$. Note that $A \subset \tilde{A}$. Since $p_{i} \circ g=f_{i}$, then $A^{\prime}=D \cap f_{i}^{-1}\left(p_{i}(A)\right)=g_{0}^{-1}(\tilde{A}) \subset g_{0}^{-1}(B)=B_{0}$.

We wish to show that $\tilde{f}_{0} \circ g_{0}\left|A^{\prime}: A^{\prime} \rightarrow\right| N \mid$ is homotopic to $h \circ f_{i}\left|A^{\prime}: A^{\prime} \rightarrow\right| N \mid$. Note that since $h \circ p_{i} \mid B \simeq \tilde{f}_{0}$, then $h \circ p_{i}\left|B \circ g_{0}\right| A^{\prime}$ is homotopic to $\tilde{f}_{0} \circ g_{0} \mid A^{\prime}$ as maps of $A^{\prime}$ to $|N|$. But $h \circ p_{i}\left|B \circ g_{0}\right| A^{\prime}=h \circ f_{i} \mid A^{\prime}$, which gives us the desired homotopy.

Since $\tilde{f}_{0} \circ g_{0} \mid A^{\prime}$ is homotopic to $F \mid A^{\prime}$ and of course $F$ is a map of $D$ to $|N|$, then $h \circ f_{i} \mid A^{\prime}$ extends to a map $F^{\prime}: D \rightarrow|N|$.

Consider the closed subset $p_{i}(A)$ of $Z_{i}$. Then for any $x \in A^{\prime}=D \cap f_{i}^{-1}\left(p_{i}(A)\right)$, $F^{\prime}(x)=h\left(f_{i}(x)\right)$. So we apply $(3)$ to get a map $H: f_{i+1}(D) \rightarrow|N|$ such that $H(z)=h\left(p_{i i+1}(z)\right)$ for all $z \in f_{i+1}(D) \cap p_{i i+1}^{-1}\left(p_{i}(A)\right)$. If $a \in A$, then $p_{i+1}(a)=$ $p_{i+1}(g(d))=f_{i+1}(d)$ for some $d \in D$. Our proof will be complete if we can show that $H \circ p_{i+1}|A: A \rightarrow| N \mid$ is homotopic to $f_{0}: A \rightarrow|N|$. Clearly, $p_{i+1}(a) \in p_{i i+1}^{-1}\left(p_{i}(A)\right)$ 
since $p_{i i+1} \circ p_{i+1}=p_{i}$. Therefore, $H \circ p_{i+1}(a)=h \circ p_{i i+1} \circ p_{i+1}(a)=h \circ p_{i}(a)$, and $h \circ p_{i}\left|A \simeq \tilde{f}_{0}\right| A=f_{0}$.

Having obtained (c) of 1.1, it remains only to prove part (d). Let $\mathcal{S}$ be a sequence of open covers of $Z$ such that $g^{-1}(\mathcal{S}) \mid D$ has a C-refinement say $\mathcal{C}$ in $D$. By Lemma 2.4 we may as well assume that $\mathcal{C}$ is finite.

It is then not difficult to show that there exists $j$ so large that for each $i \geq j$ there is a sequence $\mathcal{T}$ of open covers of $Z_{i}$ such that $p_{i}^{-1}(\mathcal{T})$ refines $\mathcal{S}$ and $\mathcal{C}$ refines $f_{i}^{-1}(\mathcal{T}) \mid D$. Choose $i \geq j$ so that $k=\gamma(i)$ and let $\mathcal{T}$ be a sequence of open covers of $Z_{i}$ as just indicated. By the construction, the sequence $p_{i i+1}^{-1}(\mathcal{T}) \mid f_{i+1}(D)$ has a C-refinement $\mathcal{D}$ in $f_{i+1}(D)$. Thus $p_{i+1}^{-1}(\mathcal{D}) \mid g(D)$ is a C-refinement of $\mathcal{S} \mid D$, so our proof is complete.

Now let us prepare for a proof of Lemma 2.2 .

2.5. Definition. Let $Y$ be a compact Hausdorff space. A triple $(A, h, N)$ will be called allowable for $Y$ if $A$ is a closed subspace of $Y, N$ is a finite simplicial complex, and $h: A \rightarrow|N|$ is a map. An indexed family $\mathcal{F}=\left\{\left(A_{\alpha}, h_{\alpha}, N_{\alpha}\right)\right\}_{\alpha \in \Sigma}$ of allowable triples is said to be dense for $Y$ if for each closed subspace $A$ of $Y$, closed neighborhood $G$ of $A$ in $Y$, finite simplicial complex $N$, and map $h: G \rightarrow|N|$, there is an $\alpha \in \Sigma$ such that $A \subset A_{\alpha} \subset G$, and a simplicial isomorphism $\Phi_{\alpha}: N \rightarrow N_{\alpha}$ having the property that $\Phi_{\alpha} \circ h \mid A_{\alpha} \simeq h_{\alpha}$ as maps of $A_{\alpha}$ to $\left|N_{\alpha}\right|$.

We give the next lemma without proof.

2.6. Lemma. Each compact Hausdorff space $Y$ has a dense family $\mathcal{F}=$ $\left\{\left(A_{\alpha}, h_{\alpha}, N_{\alpha}\right)\right\}_{\alpha \in \Sigma}$ such that card $\Sigma \leq \max \left\{w Y, \aleph_{0}\right\}$.

Proof of Lemma 2.2. Applying the above Lemma 2.6, fix a dense family $\mathcal{F}=$ $\left\{\left(A_{\alpha}, h_{\alpha}, N_{\alpha}\right)\right\}_{\alpha \in \Sigma}$ for $Z_{1},\left|N_{\alpha}\right| \subset Q_{\alpha}=Q$, the Hilbert cube, having the property that card $\Sigma \leq \max \left\{w Z_{1}, \aleph_{0}\right\}$, and for each $\alpha \in \Sigma$, let $D_{\alpha}=D \cap f_{1}^{-1}\left(A_{\alpha}\right)$. Denote by $\Sigma^{\prime}$ the set of indexes $\alpha \in \Sigma$ such that the map

$$
h_{\alpha} \circ f_{1}\left|D_{\alpha}: D_{\alpha} \rightarrow\right| N_{\alpha} \mid
$$

extends to a map $g_{\alpha}: X \rightarrow Q_{\alpha}$ with $g_{\alpha}(D) \subset\left|N_{\alpha}\right|$.

Fix also a family $\mathcal{N}$ of open subsets of $Z_{1}$ such that card $\mathcal{N} \leq \max \left\{w Z_{1}, \aleph_{0}\right\}$ and for every closed subset $A$ of $Z_{1}$ and neighborhood $G$ of $A$, there exists an element $U$ of $\mathcal{N}$ such that $A \subset U \subset G$. Denote by $\mathcal{S}_{\mathcal{N}}$ the family of finite sequences of finite covers of $Z_{1}$ by elements of $\mathcal{N}$. Clearly card $\mathcal{S}_{\mathcal{N}} \leq \max \left\{w Z_{1}, \aleph_{0}\right\}$.

Let $\mathcal{S}_{\mathcal{N}}^{\prime}$ consist of all $\mathcal{S} \in \mathcal{S}_{\mathcal{N}}$ such that $f_{1}^{-1}(\mathcal{S}) \mid D$ has a C-refinement in $D$. For $\mathcal{S} \in \mathcal{S}_{\mathcal{N}}^{\prime}$, let $\mathcal{C}_{\mathcal{S}}$ be a finite C-refinement of $f_{1}^{-1}(\mathcal{S}) \mid D$ in $D$ such that the (finite) families of $\mathcal{C}_{\mathcal{S}}$ consist of $G_{\delta}$-sets.

For $\mathcal{S} \in \mathcal{S}_{\mathcal{N}}^{\prime}$, the family $\mathcal{M}=\bigcup \mathcal{C}_{\mathcal{S}}$ is finite. For each nonempty $A \in \mathcal{M}$, take a map $f_{A}: X \rightarrow[0,1]$ such that $f_{A}^{-1}\left(f_{A}(A)\right)=A$ and let $f_{\mathcal{S}}=\prod\left\{f_{A} \mid A \in \mathcal{M}\right\}$ : $X \rightarrow[0,1]^{\text {card } \mathcal{M}} \subset Q$. Then for every $A \in \mathcal{M}$ we have, $f_{\mathcal{S}}^{-1}\left(f_{\mathcal{S}}(A)\right)=A$.

Define $\left.Z_{2}^{\prime}=Z_{1} \times \prod\left\{Q_{\alpha} \mid \alpha \in \Sigma^{\prime}\right\} \times \prod\left\{Q_{\mathcal{S}}=Q \mid \mathcal{S} \in \mathcal{S}_{\mathcal{N}}^{\prime}\right\}\right)$. Put $f_{2}^{\prime}=f_{1} \times$ $\left(g_{\alpha}\right)_{\alpha \in \Sigma^{\prime}} \times\left(f_{\mathcal{S}}\right)_{\mathcal{S} \in \mathcal{S}_{\mathcal{N}}^{\prime}}: X \rightarrow Z_{2}^{\prime}$. Let $Z_{2}=f_{2}^{\prime}(X) \subset Z_{2}^{\prime}$ and set $f_{2}=f_{2}^{\prime}: X \rightarrow$ $Z_{2}$. Denote $p: Z_{2} \rightarrow Z_{1}$ to be the restriction of the projection of $Z_{2}$ to its first coordinate. Surely $f_{2}$ is surjective and $p \circ f_{2}=f_{1}$.

We have to show that $\left(f_{1}, f_{2}, p\right)$ is lifting for simplicial complexes relative to $D$. Let $A$ be closed in $Z_{1}, N$ be a simplicial complex and $h: A \rightarrow|N|, F: D \rightarrow|N|$ be maps having the property that $F(x)=h\left(f_{1}(x)\right)$ for all $x \in D \cap f_{1}^{-1}(A)$. If 
necessary, replace $N$ by a finite subcomplex whose polyhedron contains the image of $F$. Extend $h$ to a map $h_{0}: G \rightarrow|N|$ where $G$ is a closed neighborhood of $A$ in $Z_{1}$. By virtue of Lemma 2.3, we may assume that $F\left|G_{0}: G_{0} \rightarrow\right| N \mid$ is homotopic to $h_{0} \circ f_{1}\left|G_{0}: G_{0} \rightarrow\right| N \mid$, where $G_{0}=D \cap f_{1}^{-1}(G)$.

The family $\mathcal{F}$ is dense, so there exist $\alpha \in \Sigma$ with $A \subset A_{\alpha} \subset G$ and a simplicial isomorphism $\Phi_{\alpha}: N \rightarrow N_{\alpha}$ such that $\Phi_{\alpha} \circ h_{0} \mid A_{\alpha} \simeq h_{\alpha}$ as maps of $A_{\alpha}$ to $\left|N_{\alpha}\right|$. Let $A_{0}=D \cap f_{1}^{-1}\left(A_{\alpha}\right) \subset G_{0}$. Then $F\left|A_{0}: A_{0} \rightarrow\right| N \mid$ is homotopic to $h_{0} \circ f_{1} \mid A_{0}: A_{0} \rightarrow$ $|N|$. Since $F$ is defined on all of $D$, then $h_{0} \circ f_{1} \mid A_{0}$ extends to a map $\tilde{F}: D \rightarrow|N|$.

The map $\Phi_{\alpha} \circ \tilde{F}\left|A_{0}=\Phi_{\alpha} \circ h_{0} \circ f_{1}\right| A_{0}: A_{0} \rightarrow\left|N_{\alpha}\right|$ is homotopic to $h_{\alpha} \circ f_{1} \mid A_{0}$ : $A_{0} \rightarrow\left|N_{\alpha}\right|$. Since $\Phi_{\alpha} \circ \tilde{F} \mid A_{0}$ extends to a map of $D$, so does $h_{\alpha} \circ f_{1} \mid A_{0}$. This shows that $\alpha \in \Sigma^{\prime}$, and $h_{\alpha} \circ f_{1} \mid A_{0}$ extends to the map $g_{\alpha}|D: D \rightarrow| N_{\alpha} \mid$.

Let $E=p^{-1}(A) \cap f_{2}(D)$. Define $G_{\alpha}: Z_{2} \rightarrow\left|N_{\alpha}\right|$ to be the restriction of the $\alpha$ coordinate projection of $Z_{2}^{\prime}$. Suppose that $z \in E$; then there exists $t \in D$ such that $f_{2}^{\prime}(t)=f_{2}(t)=z$. Then of course $p(z)=p\left(f_{2}(t)\right)=f_{1}(t)$, so $t \in D \cap f_{1}^{-1}(A) \subset A_{0}$. This shows that $h_{\alpha}(p(z))=h_{\alpha}\left(f_{1}(t)\right)=g_{\alpha}(t)$. On the other hand, $z=f_{2}(t)=$ $\left(f_{1}(t),\left(g_{\alpha}(t)\right)_{\alpha \in \Sigma^{\prime}},\left(f_{\mathcal{S}}(t)\right)_{\mathcal{S} \in \mathcal{S}_{\mathcal{N}}^{\prime}}\right)$ so $G_{\alpha}(z)=g_{\alpha}(t)=h_{\alpha}(p(z))$.

This helps one see that $\Phi_{\alpha}^{-1} \circ h_{\alpha} \circ p|E: E \rightarrow| N \mid$ extends to a map of $f_{2}(D)$ to $|N|$. The last step of this part of the proof is to use the fact that $\Phi_{\alpha}^{-1} \circ h_{\alpha} \circ p|E: E \rightarrow| N \mid$ is homotopic to $h \circ p|E: E \rightarrow| N \mid$, so the desired map $H: f_{2}(D) \rightarrow|N|$ extending the latter exists.

Lastly we are to show that $\left(f_{1}, f_{2}, p\right)$ is C-lifting relative to $D$. Let $\mathcal{S}^{\prime}$ be a sequence of open covers of $Z_{1}$ such that $f_{1}^{-1}\left(\mathcal{S}^{\prime}\right) \mid D$ has a C-refinement in $D$. It is not difficult to see that there is an element $\mathcal{S}$ of $\mathcal{S}_{\mathcal{N}}^{\prime}$ so that each element of $\mathcal{S}$ refines the corresponding element of $\mathcal{S}^{\prime}$ and $f_{1}^{-1}(\mathcal{S}) \mid D$ has a C-refinement in $D$. Then as provided above, $\mathcal{C}_{\mathcal{S}}$ is such a C-refinement and if $\mathcal{M}=\bigcup \mathcal{C}_{\mathcal{S}}$, then $f_{\mathcal{S}}^{-1}\left(f_{\mathcal{S}}(A)\right)=A$ for all nonempty $A \in \mathcal{M}$.

It will be sufficient to show that $p^{-1}(\mathcal{S}) \mid f_{2}(D)$ has a C-refinement in $f_{2}(D)$. We claim that $f_{2}\left(\mathcal{C}_{\mathcal{S}}\right)$ satisfies this requirement.

First, if $B \in \bigcup \mathcal{S}$, then the surjectivity of $f_{2}$ and the fact that $p \circ f_{2}=f_{1}$ can be used to show that $p^{-1}(B)=f_{2}\left(f_{1}^{-1}(B)\right)$. Hence if $A \in \mathcal{M}$ and $A \subset f_{1}^{-1}(B)$, then $f_{2}(A) \subset p^{-1}(B)$. This takes care of the refinement part. If $A^{\prime} \in \mathcal{M}$ and $A \cap A^{\prime}=\emptyset$, then we claim that $f_{2}(A) \cap f_{2}\left(A^{\prime}\right)=\emptyset$. To see this, suppose that $a \in A, a^{\prime} \in A^{\prime}$ and $f_{2}(a)=f_{2}\left(a^{\prime}\right)$. Then $f_{\mathcal{S}}(a)=f_{\mathcal{S}}\left(a^{\prime}\right)$, so $a^{\prime} \in f_{\mathcal{S}}^{-1}\left(f_{\mathcal{S}}(A)\right) \cap A^{\prime}=A \cap A^{\prime}=\emptyset$, a contradiction. This proves the necessary discreteness.

For completeness, let us state the generalizations of the (expansion) Theorems 1 and 3 of [Ma1] (resp., Theorems 5 and 6 of [Ma2]). As indicated in 4.2 of Ma2 (and see the limit theorem of $[\mathrm{Ru}]$ ), these follow from what we have done here and from the techniques of Ma1].

2.7. Theorem. Let $X$ be a compact Hausdorff space. Then there exists an inverse system $\mathbf{Q}=\left(Q_{b}, q_{b b^{\prime}}, B\right)$ of metrizable compacta $Q_{b}$ with $\operatorname{card}(B) \leq w(X)$ and $\lim \mathbf{Q}$ homeomorphic to $X$. If $K$ is a $C W$-complex and $X \tau K$, then $Q_{b} \tau K$ for each $b \in B$.

2.8. Theorem. Let $X$ be a compact Hausdorff space. Then there exists a wellordered inverse system $\mathbf{Q}=\left(Q_{\beta}, q_{\beta} \beta^{\prime}, \beta<\alpha\right), \alpha=w X$, where $X$ is homeomorphic to $\lim \mathbf{Q}$ and for each $\beta<\alpha, w Q_{\beta} \leq \beta<\alpha$, and $Q_{\beta}=\lim \left(Q_{\tau}, q_{\tau \tau^{\prime}}, \tau<\beta\right)$ if $\beta$ is a limit ordinal (in that case, $q_{\tau \beta}$ is the corresponding projection). If $K$ is a $C W$-complex and $X \tau K$, then $Q_{\beta} \tau K$ for each $\beta<\alpha$. 


\section{REFERENCES}

[Ch] A. Chigogidze, Cohomological dimension of Tychonoff spaces, Topology and its Appls. 79 (1997), 197-228. MR 99f:55003

[DR] T. Dobrowolski and L. Rubin, The hyperspaces of infinite-dimensional compacta for covering and cohomological dimension are homeomorphic, Pacific Journal of Math. 164 (1994), 15-39. MR 95a:54025

[Dr] A Dranishnikov, The Eilenberg-Borsuk theorem for mappings into an arbitrary complex, Russian Acad. Sci. Sb. Math. 81 No. 2 (1995), 467-475. MR 95j:54028

[DD] A Dranishnikov and J. Dydak, Extension dimension and extension types, Trudy Mat. Inst. Steklov. 212 (1996), 61-94. MR 99h:54049

[Dy1] J. Dydak, Cohomological dimension and metrizable spaces. II, Trans. Amer. Math. Soc. 348 (1996), 1647-1661. MR 96h:55001

[Dy2] J. Dydak, Cohomological dimension theory, Handbook of General Topology, Elsevier, Amsterdam, to appear.

[DM] J. Dydak and J. Mogilski, Universal cell-like maps, Proc. Amer. Math. Soc. 122 (1994), 943-948. MR 95a:55003

[En1] R. Engelking, General Topology, PWN-Polish Scientific Publishers, Warsaw, 1977. MR 58:18316b

[En2] R. Engelking, Theory of Dimensions Finite and Infinite, Heldermann Verlag, Lemgo, Germany, 1995. MR 97j:54033

[Ma1] S. Mardešić, On covering dimension and inverse limits of compact spaces, Illinois J. of Math. 2 (1960), 278-291. MR 22:8320

[Ma2] S. Mardešić, Factorization theorems for cohomological dimension, Topology and its Appls. 30 (1988), 291-306. MR 90a:55004

[Na] K. Nagami, Dimension Theory, Academic Press, New York, 1970. MR 42:6799

[Pa] B. Pasynkov, A factorization theorem for cohomological dimensions of mappings, Moscow Univ. Math. Bull 46 (1991), 26-31. MR 94g:54024

[Ru] L. Rubin, Cohomological dimension and approximate limits, Proc. Amer. Math. Soc. 125 (1997), 3125-3128. MR 98g:55001

[RS] L. Rubin and P. Schapiro, Compactifications which preserve cohomological dimension, Glasnik Mat. 28(48) (1993), 155-165. MR 95g:54029

Department of Mathematics, Tulane University, New Orleans, Louisiana 70118

E-mail address: mlevin@mozart.math.tulane.edu

Current address: Department of Mathematics, Texas Tech University, Lubbock, Texas 79409

Department of Mathematics, The University of Oklahoma, 601 Elm Avenue, Room 423, Norman, Oklahoma 73019

E-mail address: lrubinœou.edu

Department of Mathematics, Langston University, Langston, Oklahoma 73050

E-mail address: pjschapiro@lunet.edu 